\title{
Interdependencia y desarrollo nacional
}

\author{
Luciano Tomassini
}

Los estudios realizados en América Latina en torno al fenómeno de la transnacionalización son todavía relativamente escasos ${ }^{1}$. Además, Ia mayoría de esos estudios se ha concentrado en eI análisis del impacto de este proceso sobre las sociedades y las economías nacionales, sin influir suficientemente en los enfoques a partir de los cuales se examinan sus relaciones internacionales. Se han concentrado también en los aspectos económicos que presenta ese proceso. Por último, se han detenido en el diagnóstico del fenómeno y en la denuncia de sus consecuencias, sin avanzar suficientemente hacia el análisis de las opciones que enfrentan los países latinoamericanos en un escenario externo transnacionalizado.

Desde el punto de vista de las relaciones internacionales, hacen falta estudios que efectúen tres aportes principales: 1) Situar el análisis del proceso de transnacionalización en la base de los estudios internacionales que hoy se realizan en los países latinoamericanos y que hasta ahora han estado dominados por enfoques tradicionales; 2) Acentuar, en el examen de dicho proceso, la interrelación existente entre sus diversas facetas: económica, política, cultural y estratégica, y 3) Examinar el cambio que están experimentando las relaciones internacionales de los países en desarrollo dentro de este nuevo escenario, con énfasis en la experiencia de los países latinoamericanos, así como también el balance de riesgos y oportunidades que actualmente enfrentan dentro de un marco transnacionalizado y las condiciones bajo las cuales todavía podría ser factible maximizar su autonomía.

"Ver, sin embargo, los trabajos del xLET sobre este fenómeno, primeramente en el campo de las comunicaciones, y más adelante en relación con la transnacionalización de varios sectores en determinadas economías nacionales. Ver también los trabajos pioneros de Osvaldo Sunkel sobre la materia, tales como Capitalismo Transnacional y Desintegración Nacional en América Latina, en Estudios Internacionales No 16, enero-marzo 1971, asi como el trabajo del mismo autor con E. Fuenzalida, titulado Capitalismo Transnacional y Desarrollo Nacional, en el No 44 de la misma publicación. 


\section{El marco de referencia general.}

Tradicionalmente se entendió que las relaciones internacionales incluían las relaciones mantenidas entre estados soberanos a través de sus fronteras nacionales. Hoy tienden a concebirse en forma más amplia, como toda relación que se establezca entre varias sociedades nacionales, a través de diferentes grupos y en función de diversos intereses. Se acrecienta la interpenetración entre las sociedades nacionales. La agenda internacional se vuelve más compleja. El estado deja de monopolizar el manejo de las relaciones externas y nuevos agentes comienzan a intervenir en ellas.

Muchos factores han determinado esta fundamental transformación. En el plano internacional, el mundo rígidamente jerarquizado que emergió de la postguerra cede paso a un mundo multipolar, con la reconstrucción o el surgimiento de Europa, el Japón y China y con la incorporación a la comunidad internacional de un creciente número de países en desarrollo, muchos de los cuales disponen de un margen de maniobra apreciablemente ampliado. En el frente interno, los estados nacionales se comprometen con una gama de objetivos cada vez más amplia y más compleja, que junto a la seguridad nacional incluyen el desarrollo económico, la elevación del ingreso, la defensa del empleo, la preservación del medio ambiente, la identidad cultural y la calidad de la vida en sus respectivas sociedades, objetivos que pasan a gravitar decisivamente en sus relaciones externas. Se transita de un mundo dominado por consideraciones de seguridad estratégica y por la confrontación entre las dos superpotencias, como fue el mundo de postguerra, a otro caracterizado por la búsqueda de la distensión y por una atmósfera más favorable a la prosecución de otros intereses -económicos, tecnológicos, sociales, ecológicos y culturales- en las relaciones entre las distintas naciones.

Esta visión moderna de las relaciones internacionales se diferencia substancialmente de la visión clásica o "realista", que ha prevalecido hasta ahora, centrada en consideraciones vinculadas con la esfera de la seguridad, y a lo que dio en llamarse la "política del poder" en las relaciones entre los estados. Greemos que esa visión ha enriquecido considerablemente el estudio de las relaciones internacionales. En otro lugar he señalado que "el análisis de la política internacional concebida exclusivamente en términos de poder tiende a vaciar la interpretación de todo contenido substantivo -a perder de vista los objetivos en torno a los cuales se articulan las relaciones de cooperación o de conflicto- y a imprimirle un carácter ritual que la convierte, como decía Toynbee, aludiendo a Macbeth, en un cuento narrado por un idiota"'.

${ }^{2} \mathrm{~L}$. Tomassini (editor), Relaciones Internacionales de ta América Latina, México, 1981. Introducción. 
La visión "realista" se basabá en varias presunciones. La primera concebía la política internacional como centrada en torno a los intereses de las grandes potencias, con una u otra de las cuales debían alinearse los estados menores, dando lugar a la formación de bloques o esferas de influencia al interior de los cuales la potencia hegemónica dirimía conflictos e imponía cierto orden. La segunda se refería al rol del estado como actor internacional e implicaba no sólo que éste detentaba el monopolio de las relaciones internacionales, sino que actuaba en ese plano como una unidad coherente, $y$ en función de un número reducido de objetivos (generalmente vinculados con el mantenimiento de la paz y la seguridad). De allí una tercera presunción, que reducía la agenda internacional a un limitado número de temas, rígidamente jerarquizados entre sí. La cuarta presunción se refería a la básica homogeneidad de los agentes que actúan en la vida internacional, a saber (como ya se ha dicho), los estados. No es de extrañar que, en quinto Iugar, se tuviera una visión iguaImente restringida acerca del repertorio de recursos de poder que un estado podía emplear para influir sobre otros, los que parecían preferentemente circunscritos a la esfera política y militar. Por último, se presumía que las arenas en las cuales podían darse relaciones de cooperación o de conflicto entre los estados eran, en general, pocas, precisas y suficientemente conocidas. Aquella concepción dio lugar a una "diplomacia de doble vía", que reservaba a los estadistas el manejo de los asuntos incluidos dentro de los estrechos parámetros que según. las convicciones en boga enimarcaban la alta política internacional, dejando en manos de los hombres de negocios y los agregados comerciales de las embajadas la conducción de las relaciones económicas entre los estados y otras cuestiones de menor importancia ${ }^{3}$.

Todas esas presunciones han sido sobrepasadas por la evolución de la realidad internacional contemporánea. A la intemalización del comercio y de la producción primaria, que dominó la primera mitad del siglo $\mathrm{xx}$, siguió la de la producción industrial y los servicios, de tal manera que a comienzos de los años setenta el valor de la producción de las empresas transnacionales fuera de su país de origen había sobrepasado con creces el del comercio internacional, en tanto que una proporción creciente de éste se verificaba al interior de esas mismas firmas. Por esa misma época, la inconvertibilidad del dólar primeramente, los excedentes acumulados por la OPEP más tarde y la recesión en los países industrializados, por último, provocan el renacimiento de los mercados financieros internacionales, representados por los mercados de euromonedas, cuyo tamaño en la actualidad excede el trillón de dólares, superando también el valor de los activos controlados por las autoridades mone-

${ }^{3} \mathrm{R}$. Cooper, Trade Policy is Foreign Policy, en Foreign Policy No 9, 1972-73. 
taxias nacionales. Al mismo tiempo, la innovación tecnológica se convierte en el principal factor de competencia entre las mayores naciones industrializadas, en circunstancias de que su control radica fundamentalmente en las empresas transnacionales, ya sea actuando en función de sus propios planes de expansión o de contratos suscritos con el estado. El crecimiento económico y la ampliación de la democracia en las sociedades avanzadas determinan que un número cada vez mayor, más fuerte y más articulado de grupos organizados adquiera un interés directo en las consecuencias internas de acontecimientos registrados en otras sociedades, tales como cambios en los niveles relativos de productividad, avances tecnológicos, tendencias recesivas, cambios en el empleo, presiones inflacionarias, triunfos electorales de determinadas corrientes ideológicas y los consiguientes cambios de políticas. Por último, la intensidad del conflicto ideológico entre las grandes potencias, el fuerte efecto de demostración asociado a los grandes sistemas económicos y sociales en conflicto -capitalismo y socialismo- y el avance en las comunicaciones, desencadenan fuertes influencias culturales de origen transnacional sobre todas las sociedades nacionales.

Varios elementos deberían subrayarse en un esfuerzo de reelaboración teórica que pudiera dar cuenta de estas nuevas realidades.

- Ante todo, estos elementos deberían definirse a la luz de un análisis muy amplio de la actual tendencia hacia la multipolaridad, y de su impacto sobre la estructura internacional contemporánea.

-A continuación deberían analizarse los efectos del gradual compromiso del estado con un número creciente de objetivos sobre sus relaciones internacionales.

-Dentro de este contexto debería prestarse más atención al hecho de que en la actualidad las relaciones internacionales han dejado de estar circunscritas a las relaciones de estado a estado para abarcar todos los nexos que se producen entre diversas sociedades nacionales.

Puede aventurarse la hipótesis de que, a diferencia del pasado, en la actualidad las relaciones internacionales I) son protagonizadas por un creciente número de centros de poder, 2) cuya actuación externa tiende a satisfacer una gama de objetivos mucho más amplia que en el pasado, 3) se desenvuelven en torno a una agenda más compleja y menos jerarquizada, 4) son manejadas por nuevos y múltiples agentes, distintos del estado, 5) poniendo en juego recursos de poder no tradicionales, y 6 ) en una variedad de arenas mucho más numerosas, cambiantes y entrelazadas que antes.

Estos elementos de análisis permitirian reconstruir la existençia y operación de múltiples "juegos", "circuitos" o "procesos" desarrollados alrededor de la agenda, los agentes, los recursos y las arenas anteriormente mencionados, que vinculan de múltiples y diversas 
maneras a las distintas sociedades nacionales, en función de temas específicos.

Se trata, en suma, de un enfoque que busca poner de manifiesto la interpenetración que actualmente se da entre las distintas sociedades nacionales y enfatiza su sensibilidad -o vulnerabilidad como situación extrema frente al contexto externo4.

La existencia de una aguda relación de interdependencia entre todos los países del mundo, y la sensibilidad de las diversas sociedades nacionales frente al contexto externo, se han puesto claramente de manifiesto, como consecuencia de la crisis por que atraviesan las sociedades industriales, $y$ de la toma de conciencia, a nivel global, acerca de la existencia de límites al crecimiento experimentado por la economía mundial durante el período de postguerra.

El extraordinario período de auge por el que atravesaron los grandes países industrializados durante los decenios de 1950 y 1960 constituyó el telón de fondo que hizo posible la formación del sistema internacional contemporáneo -y, como veremos más adelante-, la progresiva integración en él de los países en vías de desarro1lo. Sin embargo, con el transcurso del tiempo, fueron poniéndose de manifiesto los costos, las contradicciones y los efectos indeseables de dicho proceso. El decenio de 1970 se distinguió por la inflexión del ciclo expansivo de los centros, fenómeno que determinó la evolución de la economía mundial en su conjunto, caracterizada desde entonces por la inseguridad en el abastecimiento de energía y de otras materias primas estratégicas, por una situación de inestabilidad aguda y por tendencias inflacionarias y recesivas crónicas. De allí que a lo largo del último período no haya cesado de profundizarse la conciencia de que el crecimiento económico tiene ciertos límites. El primer informe publicado bajo los auspicios del Glub de Roma sobre esta problemática contribuyó a iniciar un debate que generó una pluralidad de reacciones, en el plano teórico, mientras que las decisiones adoptadas por la OPEP en 1973 dieron la señal de alarma en el terreno de las realidades ${ }^{5}$.

En el plano económico, esta conciencia está asociada a las tendencias observables actualmente en los centros, entre las cuales destacan el aumento de los costos de operación de sus sistemas productivos, como consecuencia de la elevación de los salarios y del gasto público, la declinación de la productividad, la caída de las inversio-

${ }^{1}$ Ver R. Cooper, The Economics of Interdependence, 1968; R. Keohane y J. Nye, Buyer and Interdependence: World Politics in Transition, 1977; Klaus Norr y Frank N. Trager (editores), Economic Issues and National Security, 1977; R. Muller, Revitalizing America, Politics for Prosperity, 1981, etc.

${ }_{M}$ Leadows et al., The Limit to Growth, 1972; B. Ward, Only One Earth, 1973; L. Brown, WorId Without Borders, 1973; N. Mesarovic y E. Pestel, The Mankind at the Turning Point, 1974; A. Herrera y otros, Catastrophe or New Society, 1976; E. J. Mishan, The Economic Growth Debate: An Assessment, 1977, etc. 
nes, la disminución de Ia rentabilidad de las mismas, la atenuación del ritmo de innovación tecnológica y, en general, la pérdida de competitividad en un creciente número de actividades productivas, en un contexto de fuertes presiones medio ambientales y en un clima generalizado de inseguridad en cuanto al abastecimiento de energía y otras materias primas industriales.

Desde un punto de vista socio-político se ha señalado la tendencia hacia la oligopolización de las relaciones sociales que caracteriza a las sociedades avanzadas y la consiguiente pugna redistributiva como un factor de rigidez y crisis del sistema. Los cambios que han tenido lugar en la estructura de la fuerza de trabajo en dichas sociedades como consecuencia de la mayor diferenciación y calificación de sus distintos segmentos, de la mutación en las preferencias de los trabajadores y de la protección otorgada a ciertos sectores por las organizaciones sindicales o por políticas gubernamentales, contribuyen a fortalecer esas rigideces. La colusión entre agrupaciones de productores que buscan ejercer conjuntamente su poder económico o político para posponer innovaciones o impedir cambios en la asignación de recursos que puedan amenazar sus posiciones, postergando el ajuste que deberían encarar esas economías para adecuarse a las nuevas condiciones creadas por el impacto de factores externos en un mundo interdependiente, es una fuente adicional de rigideces. Entre ellas se cuenta también el crecimiento de la intervención gubernamental a través de la proliferación de medidas reguladoras, el aumento del gasto público, o la creciente participación del estado en proyectos industriales por razones estratégicas o socio-políticas.

Una última categoría de factores que está incidiendo en la transformación y crisis del sistema transnacional contemporáneo se refiere a la profunda xautación de los valores o preferencias sociales que está experimentando un número creciente de grupos y sectores en el interior de las sociedades avanzadas. Desde la "sociedad postindustrial" hasta la sociedad "hecha a la medida" que podría suceder a la era de producción masiva, pasando por el ataque al gigantismo de los sistemas económicos y sociales prevalecientes en los países industriales lanzado bajo el slogan de "small is beautiful", son numerosas las imágenes que la ciencia social de los países nórdicos nos ofrecen para interpretar su transición y crisis. Según un informe de la OCDE, esa transición se expresaría en el reemplazo de un conjunto de valores materialistas por otros de carácter post-materialista que giran en torno a preferencias vinculadas con la calidad de la vida ${ }^{6}$.

Ver D. Bell, The Coming of Post-Industrial Society, 1973, y The Cultural Contradictioss of Capitalism. 1976; A. Toffler, The New Wave, 1980; E. F. Schumacher, Small is Beautiful: Economics as if People Mattered, 1973, OCDE, Facing the Future, 1979, etc. 
La crisis de las sociedades industriales, el recrudecimiento de la competencia entre ellas con las consiguientes amenazas neomercantilistas, y la transmisión internacional de las perturbaciones económicas, primeramente; la mayor sensibilidad de un creciente número de grupos sociales organizados frente al impacto de factores externos, en seguida; la internacionalización y pugna de ideologías y concepciones del mundo contrapuestas, por último, han agudizado la sensibilidad de las diversas sociedades nacionales frente al contexto externo. Ello subraya la necesidad de analizar el proceso de transnacionalización $\mathrm{y}^{r}$ su principal resultado: el incremento de la interdependencia, así como el impacto de esta tendencia sobre la evolución de las sociedades nacionales, con énfasis en la experiencia de los países en vías de desarrollo.

\section{Las relaciones internacionales de los paises en desarrollo}

En diversos lugares hemos señalado el cambio que han experimentado las relaciones internacionales de los países en desarrollo a lo largo de los últimos treinta años ${ }^{7}$.

Durante la segunda postguerra se acentúa el interés de la comunidad internacional por los países subdesarrollados. Como consecuencia de ello surge una serie de teorías sobre la naturaleza del desarrollo y sobre las estrategias más adecuadas para promoverlo. El común denominador de todos esos enfoques radica en su tendencia a asimilar "desarrollo" con "modernización". El desarrollo era concebido como un proceso unívoco, que pasa necesariamente por etapas preestablecidas, siguiendo un camino que deben recorrer de un modo similar todos los países. Apenas se planteaba la posibilidad de que la causa del subdesarrollo de los países periféricos radicara en sus relaciones con los grandes centros industriales. Se presumía una suerte de "armonía natural de intereses" entre ambos grupos de países. El desarrollo de los países periféricos debía.ser inducido por el crecimiento económico del mundo industrializado con el complemento de programas de cooperación internacional impulsados por éstos. De allí el papel central que en dicha etapa ocupara la "ayuda externa". Pero así como las estrategias de desarrollo basadas en el concepto de "modernización" no condujeron a los resultados esperados, en el plano interno, en el plano internacional el período que se extiende hasta los años 60 concluyó con un acendrado sentimiento de "desilusión frente a la ayuda".

TO. Sunkel y L. Tomassini, La Crisis del Sistema Transnacional y la Transformación de las Relaciones Internacionales de los Países en Desarrollo, en Estudios Internacionales $N$ P 50, abril-julio de 1980. Ver también L. Tomassini, Factores Ambientales, Crisis en los Centros y. Cambio en las Relaciones Internacionales de América, en Revista de la GEPAL No 12, enero de-1980. 
El enfoque anterior tenía, entre otros, el defecto de ser profundamente ahistórico. Pasaba por alto los antecedentes y las características estructurales que moldearon durante largo tiempo las relaciones entre los países en desarrollo y desarrollados. Suponía que el desarrollo constituye un proceso que se da independientemente en distintos lugares y momentos del tiempo y que incluso consiste en reproducir, bajo distintas circunstancias, un modelo previo. La GEPAL cuestionó estas presunciones desde comienzos de los años 50 a través del esquema de análisis centro-periferia. Dicho análisis contuvo, desde un principio, los elementos que, andando el tiempo, llevarían a plantear la existencia de un sistema económico que genera a la vez desarrollo en los centros y subdesarrollo en la periferia. Ese mecanismo se basaba è una división internacional del trabajo en que a la periferia correspondía el papel de producir alimentos y materias primas para los grandes centros industriales, importando desde éstos los bienes manufacturados necesarios para atender sus necesidades de consumo, así como también los bienes de capital e intermedios requeridos para proseguir su proceso de desarrollo. La declinante relación de los precios de intercambió entre ambos grupos de productos, en contra de las exportaciones de los países periféricos, explica su secular estancamiento. Pero las estrategias fundadas en ese diagnóstico -la industrialización por sustitución de importaciones, destinada a alterar la especialización de los países periféricos en la economía internacional, y la regulación de los mercados de productos básicos- no mejoraron su situación sensiblemente.

Entretanto, el escenario internacional cambió en forma dramática, como consecuencia del proceso de transnacionalización señalada más arriba y del aumento de la interdependencia. Dentro de este contexto, cambió la naturaleza de los problemas en torno a los cuales giran las relaciones internacionales de los países en desarrollo: a los temas de los productos básicos, la inversión extranjera y la ayuda externa se han agregado la preocupación por la energía, el proteccionismo y el acceso a los mercados de los países industrializados, el redespliegue industrial, la fluidez y predictabilidad de los mercados financieros internacionales, la capacidad de absorber y adaptar tecnologías externas, el mejoramiento de las formas de contratación con las corporaciones transnacionales, la preservación del equilibrio ecológico y la defensa de la identidad cultural de esos países. También han cambiado los agentes del proceso, antiguamente representado por los gobiernos de Ios países industrializados, las compañías transnacionales radicadas en los sectores primarios y las instituciones financieras internacionales, añadiéndose las empresas localizadas en los sectores manufactureros y de los servicios y otros protagonistas representados por agrupaciones intelectuales, religiosas, laborales, políticas y militares de la más diversa naturaleza. 
En suma, como consecuencia del avance de la transnacionalización se ha transformado el patrón de relaciones centro-periferia que prevalecía durante el período de postguerra. En otras palabras, si bien el esquema centro-periferial continúa siendo válido como categoría de análisis, debe aplicarse a una estructura internacional muy diferente a la del pasado. Ello exige una reinterpretación de las relaciones entre los dos segmentos del sistema, a la luz de la cual probablemente los centros presenten una imagen menos monolítica y algunos países en desarrollo ocupen una posición menos periférica.

Por cierto que esta transformación no afecta por igual a todos los países de la periferia. Tan importantes como las grandes modifícaciones experimentadas por los centros durante el último decenio, para explicar el cambio que ha tenido lugar en las relaciones internacionales de los países en desarrollo, son las transformaciones que durante ese mismo período han experimentado algunos de ellos. Como consecuencia de esas transformaciones ha surgido, en medio de enconados debates conceptuales, una categoría de países de "desarrollo intermedio" que, manteniendo muchos problemas en común con el resto de los países en desarrollo, se distinguen de ellos desde el punto de vista de su grado de desarrollo económico, la amplitud y diversificación de su base industrial, su capacidad para incorporar y adaptar tecnología externa, la participación de las manufacturas en sus exportaciones globales ( $y$ de una creciente gama de productos complejos dentro de las primeras), su acceso a los mercados financieros internacionales, su creciente habilidad para contratar con las empresas transnacionales, la calificación de su fuerza de trabajo, sus niveles de educación y el grado de articulación de sus sociedades civiles, entre otros factores ${ }^{S}$.

"No era posible realizar esos cambios en forma repentina -señala el Informe Brandt refiriéndose a esas transformaciones- pero descle los años 60 muchos países en desarrollo evolucionaron hacia estrategias encaminadas a promover las exportaciones y a compensar las desventajas derivadas del aislamiento de sus mercados nacionales. Gran número de países que introdujeron políticas orientadas hacia las exportaciones, fueron capaces de explotar sus ventajas comparativas en los mercados mundiales. Entre ellos se incluyen algunos países latinoamericanos con una relativamente larga historia de independencia nacional y algunas islas y ciudades-estado cuyas economías se vieron obligadas desde un comienzo a depender de la demanda externa. Una vez que la industrialización hubo echado raíces, no sólo las industrias intensivas en mano de obra, como la del vestuario y el cuero, sino también otras moderadamente inten-

\footnotetext{
sVer capítulo sobre los países de desarxollo intermedio en el volumen editado por E. Hill y L. Tomassini, bajo el título América Latina y el Nuevo Orden Económico Internacional.
} 
sivas en capital, como la electrónica, la del acero y la de la cons. trucción de barcos, se han tornado altamente competitivas en los mercados internacionales".

Es cierto que los beneficios derivados de este excepcional período de crecimiento económico se distribuyeron en forma muy desigual entre los distintos grupos de países en desarrollo, y que aquellos que ya han alcanzado una etapa de desarrollo intermedio en América Latina y en el Sudeste de Asia (los que aplicando un indicador muy restrictivo, el Banco Mundial denomina de "ingresos medios" y, en forma más restrictiva aún, la literatura anglosajona denomina newly industrializing countries o NIG's) tienden a absorber una proporción desmesuradamente alta de los mismos, en contraste con la mayoría de los países de Asia y Africa, que han registrado tasas de crecimiento inferiores y en donde vive la mayoría de los pobres del mundo. Pero, debe tenerse presente también que el desarrollo es un proceso dinámico, y que es probable que un número cada vez mayor de países en desarrollo pueda acceder en forma paulatina a aquellas etapas intermedias, de tal manera que los paises que hoy se encuentran en un estadio relativamente más avanzazados podrían estar señalando el camino que en el futuro habrán de seguir los países menos desarrollados. Hay antecedentes para que tal presunción se plantee en el caso de América Latina.

El hecho es que las relaciones internacionales de los países en desarrollo están cambiando, en un contexto externo crecientemente transnacionalizado, aun cuando esas modificaciones se manifiesten inicialmente en diversos grados y con marcadas diferencias según el tipo de países.

\section{El sentido del cambio: algunos indicadores.}

Gonviene examinar, brevemente, para ilustrar nuestra hipótesis, algunos de los aspectos en que se manifiestan los cambios que han experimentado recientemente las relaciones internacionales de los paises en desarrollo.

En el plano económico, que es el que más se ha analizado, dichos aspectos se advierten en las nuevas formas de interdependencia ecológica, económica y financiera que tan fuertemente condicionan la evolución y perspectivas de la economía mundial contemporánea.

- Los años 70 marcaron el fin de una era caracterizada por un prolongado período de crecimiento de los centros industriales, basado en un suministro abundante y barato de energía y dependiente de un producto no renovable, como el petróleo. El hecho de que, como consecuencia del estilo de desarrollo prevaleciente en los centros industriales, la demandạ energética tendiera a crecer más rápi- 
damente que la producción de este recurso, unido al recrudecimiento de las tensiones planteadas en el Medio Oriente dentro de un escenario de mayor fluidez internacional, hicieron que los países exportadores de petróleo utilizaran su organización con el fin de ejercer presiones eficaces para elevar los precios del petróleo. Todo indica que éstos seguirán elevándose, a distintos ritmos, hasta alcanzar niveles que permitan incorporar al uso económico fuentes alternativas de energía cuya operación ès hoy más costosa, con las consiguientes presiones sobre las balanzas de pagos de los países importadores. Al mismo tiempo, a dichas presiones se añaden los requerimientos de inversión de esos países, con el objeto de desarrollar nuevas fuentes convencionales o alternativas de energía, programas que se elevarán de alrededor de doce mil millones de dólares anuales de inversiones a mediados del decenio pasado, a cincuenta mil y ochenta mil millones, respectivamente, durante el primer y segundo quinquenios de los años 80, tan sólo en el caso de América Latina. Todo ello crea una fuerte mutualidad de intereses entre los países desarrollados y en desarrollo en torno a 1) el descubrimiento y difusión de tecnologías que permitan la conservación de energía, 2) el financiamiento de los desequilibrios de balanzas de pagos y de las inversiones requeridas para mantener los mercados e incrementar la oferta energética en los países en vías de desarrollo, y 3) la puesta en común de tecnologías que permitan incorporar fuentes nuevas y renovables de energía, particularmente en el Tercer Mundo. La crisis energética provocó debate en torno a si el petróleo sería "la excepción"9 o la "cabeza del iceberg" de una situación prospectiva de escasez generalizada de alimentos y materias primas industriales. La iniciativa del Secretario de Estado Kissinger en la IV UNCTAD, en Nairobi, encaminada a establecer un banco internacional de recursos, rechazada por el Grupo de los 77, $y$ el subsiguiente esfuerzo de las naciones industrializadas por desarrollar los recursos naturales del Tercer Mundo a través de ingentes programas de inversión y nuevas formas de contratación con los países receptores, tienden a demostrar que, con altibajos, es la segunda hipótesis la que se ve como más probable.

-Esta situación de interdependencia se repite en el campo del comercio, la industrialización y la división internacional del trabajo. La participación de las manufacturas en las exportaciones totales de los países en desarrollo se ha ido ampliando desde el $10 \%$ en 1955 , al $20 \%$ diez años más tarde, y al $40 \%$ en 1975 . Se espera que ellas representen más de la mitad de las exportaciones globales del Tercer Mundo al promediar el presente decenio. Para Sir Arthur Lewis, "la división del mundo entre países en desarrollo que exportan productos agrícolas e importan manufacturas, y países de-

${ }^{\circ} \mathrm{R}$. Cooper, Oil is the Exception, en Foreign Policy. 
sarrollados que hacen lo contrario, está en vías de desaparecer" y, por lo tanto, "la supresión de las restricciones a los productos industriales importados es lo más importante que podría ocurrir en el área del comercio internacional" desde el punto de vista del Tercer Mundo 10 . Esto explica el recrudecimiento del proteccionismo, y sus nuevas modalidades, en Ios países industrializados. Entre estas modalidades se cuentan su carácter más sistemático, su permanencia y su selectividad, esto es, su aplicación discriminatoria contra aquellos productos que se van tornando más competitivos, particularmente los bienes industriales que están en condiciones de exportar los países en desarrollo. Gon todo, hay una diferencia substancial entre esta situación y aquella en que estos países se encontraban a comienzos de la postguerra, cuando simplemente no podían exportar manufacturas. Detrás del velo proteccionista levantado por los países industrializados se encuentra su pérdida de capacidad competitiva en un creciente número de ramas industriales. En tal sentido, debería constituir un fenómeno esencialmente transitorio y adverso a los intereses de los propios países avanzados, no sólo porque tiende a postergar el ajuste de sus economías, sino porque limita la expansión de los ingresos y, por consiguiente, de los mercados de los países en desarrollo, mercados que han pasado a adquirir una importancia creciente para la recuperación de los países industrializados. La redistribución industrial, esto es, la internacionalización del ciclo productivo, a través de una variedad de formas de inversión extranjera directa, acuerdos de coproducción, mecanismos de subcontratación, arreglos de comercialización o de servicios técnicos y otros, constituye una faceta complementaria de este mismo proceso, que tiende a integrar cada vez más, aunque naturalmente en forma asimétrica, los dos grandes segmentos de la economía internacional.

- Ya se ha señalado que la economía mundial durante el último decenio se caracterizó por haber alcanzado un extraordinario grado de liquidez financiera internacional. Este fenómeno tornó elegibles para la banca privada y los mercados financieros internacionales a deudores que antes no lo habrían sido, entre ellos un creciente número de países en desarrollo. Esta situación hizo posible el explosivo crecimiento de la deuda externa de estos últimos países, fenómeno que fue acompañado de un drástico cambio de su composición, en favor de obligaciones contraídas con fuentes privadas. La deuda externa de los países en desarrollo no exportadores de petróleo bordeaba los 400 mil millones de dólares hacia 1980, a lo cual debe sumarse una deuda cercana a los 100 mil millones contraída por el campo socialista, todo lo cual implica que aproximadamente la mitad del trillón de dólares (un millón de millones de dóla-

${ }^{10} \mathrm{~A}$. Lewis, The Evolution of the International Economic Order, 1979. 
res) disponibles en Ios mercados financieros internacionales a fines del año pasado se había canalizado en forma de compromisos asumidos por países que hasta entonces se encontraba virtualmente marginado del circuito financiero internacional. Debe tenerse en cuenta que, al acudir a ese grado de endeudamiento externo, los países en desarrollo estaban optando por postergar la contracción económica que habrían experimentado como consecuencia de la recesión mundial y del aumento de los precios del petróleo y de los bienes de capital. Por otra parte, también estaban incrementando su vulnerabilidad externa, como consecuencia de la estructura del nuevo endeudamiento en lo que se refiere a la elevación de sus tasas de interés, su inestabilidad y la brevedad de los plazos a que se sujetan esos compromisos. Es este, en todo caso, otro campo en que el entrelazamiento entre las economías desarrolladas y en desarrollo se pone de manifiesto.

Similares consideraciones cabe formular en el campo político:

-Ya. se ha señalado también que, como consecuencia de la ampliación de los procesos democráticos, la elevación del ingreso y el incremento del bienestar que ha tenido lugar en los países industrializados, no han cesado de fortalecerse, articularse y proyectarse en todas direcciones un creciente número de grupos de interés al interior de esas sociedades. Cada vez resulta más frecuente que, en forma organizada, esos grupos salgan en defensa de los intereses de grupos afines o se interpongan para frustrar las expectativas de sus competidores en otras sociedades internacionales. Tal es el caso no sólo de numerosos grupos económicos, sino también de otras agrupaciones sectoriales o regionales, así como de un sinnúmero de agrupaciones laborales, burocráticas o profesionales cuyos intereses dependen cada vez más de la prevalencia de ciertos valores, normas - conceptos a nivel internacional. Lo mismo ocurre en relación con la proyección externa de algunos partidos políticos, grupos religiosos o corrientes de opinión: si bien es cierto que el proceso democrático ha tendido a morigerar las posiciones ideológicas extremas al interior de esas corrientes, no es menos cierto que en general éstas han acrecentado su gravitación, vigencia y proyección externa. Tras el quebranto sufrido por las instituciones políticas después de la Segunda Guerra Mundial, y la extrema rigidez con que se manejaron las relaciones diplomáticas de estado a estado durante ese período, volvieron a reconstituirse y a emerger en el plano internacional un sinnúmero de instituciones que de maneras mur variadas expresan distintas corrientes ideológicas a través de agrupaciones intelectuales y religiosas, movimientos estudiantiles, organizaciones laborales, grupos étnicos, fundaciones, comunidades científicas, cuerpos voluntarios y otros mecanismos más o menos sutiles, que florecen con más fuerza en aquellas sociedades que poseer una tradición democrática más ideológica y articulada, como las 
europeas, que en sociedades de estructuración más reciente como la de los Estados Unidos. En otro lugar se ha señalado el resquebrajamiento de la red de instituciones y programas públicos en que se 'enmarcó el sistema internacional en la postguerra y la tendencia hacia la privatización de las relaciones intemacionales ${ }^{11}$. Es este el nuevo marco político en que se desenvuelven las relaciones externas de los países en desarrollo.

- En un plano estratégico, el efecto disuasivo causadó por la acumulación de armamentos nucleares, la necesidad de dirimir cónflictos potenciales mediante el uso de recursos convencionales; Ia consiguiente fragmentación del teatro de las operaciones, el deseo de evitar la participación en ellas de las grandes potencias y de actuar a través de los llamados proxies, y el auge de las estrategias de "respuesta flexible" (todo ello unido a la importancia estratégica adquirida por una serie de temas, actores, recursos y arenas que no tenían ninguna importancia o habían desempeñado un papel subordinado durante la etapa de la guerra fría), acentúan la interdependencia militar entre naciones de envergaduras muy dispares, $e$ introducen en la palestra mundial a un creciente número de países y regiones en desarrollo.

- Finalmente, la progresiva desilusión que se experimenta en las sociedades industriales frente al creciente papel del estado y a la proliferación de sus regulaciones, la tendencia a la privatización de las más variadas actividades, el fortalecimiento de la sociedad civil en diversas esferas $y$, en el seno del mismo gobierno, el predominio de la. "política burocrática" vis-a-vis un comportamiento supuestamente homogéneo del estado, alientan el surgimiento y presencia de múltiples agentes que intervienen con intereses y estilos propios en el manejo de las relaciones internacionales, incluso en el plano de las relaciones entre países industrializados y países en desarrollo ${ }^{12}$.

Los mismos resortes operan en el plano cultural:

-Desde las pautas de comportamiento "modernas" de Parsons -universalismo en los criterios, especificidad en Ios roles, prosecución del logro y neutralidad afectiva versus la aplicación de criterios particularistas, el desempeño de roles difusos, la adscripción a un grupo y el predominio de motivaciones afectivas- hasta su versión latinoamericana según Gino Germani -acción electiva versus acción prescriptiva- pasando por Riesman, McGlellan y Hagen, entre otros, la sociología cultural acuñada en los países industriali. zados procura difundir una racionalidad y un concepto de modernidad favorable a la movilidad social, la competencia económica, el

IITrabajo inédito de O. Sunkel y E. Lahera.

ITVer OCDE, The Welfare State in Crisis, 1981. 
lucro empresarial, la innovación tecnológica y el papel del mercado como marco fundamental de los comportamientos señalados.

-Surge así el funcionalismo como base no sólo de las ciencias sociales sino, y muy especialmente, de una cierta ingeniería social encaminada a crear un mundo seguro para la expansión de las sociedades industriales, a través de la subordinación de los distintos procesos sociales a los requerimientos planteados por la operación eficiente de un sistema poco respetuoso de todo particularismo y que, además, no postula valores cualitativos, sino que busca en su simple operatividad la justificación y garantía de su supervivencia. Todo ello se traduce en el surgimiento y la proyección de un ethos que entroniza el mercado como el supremo asignador de los "valores" sociales, que hace del éxito -medido según un patrón estrecho- la fuente del mérito, convierte a la publicidad en la principal fuente de arquetipos sociales, y orienta al grueso de las expectativas del público hacia el consumo de bienes materiales.

- Una de las consecuencias de estas teorías es la ya señalada asimilación de los conceptos de "desarrollo" y "modernización", concebida esta última como la adopción de las instituciones y pautas de los países que realizaron la revolución industrial, lo cual redunda en un proyecto encaminado hacia la construcción de un mundo seguro para el desarrollo del capitalismo a través de la expansión de las sociedades industriales.

Ha sido precisamente la profundización de esta ética $-\mathrm{y}$ su proyección transnacional a través de complejos engranajes- lo que ha suscitado al interior de las propias sociedades avanzadas movimientos contraculturales, como aquellos a que se hacía referencia más arriba.

\section{La experiencia latinoamericana}

Surgen así múltiples, nuevos y más complejos canales de vinculación entre los países en desarrollo y las sociedades avanzadas. Esta tendencia, que cada día percibimos con mayor claridad, ya fue intuida por uno de los más recordados pensadores de la CEPAL, don José Medina Echavarría, cuando planteaba "la posibilidad de que alguna vez quede anticuado el esquema actual de la dependencia, por la modificación rápida o lenta de su naturaleza, como dependencia hegemónica unilateral", y cuando sugería el surgimiento de "una forma inédita de esa dependencia como relación plurilateral"13:

Esta tendencia es registrada por el Secretario Ejecutivo de la Comisión, en su informe al xvmr período de sesiones de dicho organis-

${ }^{13} J$. Medina Echavarría, América Latina en los Escenarios Posibles de la Distensión, en Revista de la GEPAL No 6, segundo semestre de 1976. 
mo, al señalar: "La experiencia de los últimos años demuestra que América Latina ha seguido un curso de desarrollo más abierto y entrelazado con la economía internacional. Este hecho no es nuevo como a todos nos consta. En efecto, la gravitación de los factores externos ha sido una constante histórica en la región y, en general, en los países en desarrollo. Lo que sí es nuevo son las modalidades que ha adquirido este fenómeno".

Dicha tendencia se refleja, históricamente, en la aguda dependencia que ha demostrado América Latina frente a los vaivenes del ciclo externo. En efecto, durante los últimos treinta años las economías latinoamericanas han tenido un comportamiento notable, al menos en términos de crecimiento, con una tasa de expansión anual superior al $5.5 \%$-, un promedio superior al de cualquiera otra región del mundo durante esos treinta años. Ello se logró en condiciones de una aguda dependencia externa y gracias a que la región respondió en forma flexible y generalmente eficiente a las limitaciones y oportunidades creadas por el contexto externo en cada período.

Así, por ejemplo, durante los años 50 y primera mitad de los 60 , la desarticulación de la economía mundial, el énfasis en el desarrollo y la integración de los países industrializados y la tendencia al deterioro de los términos de intercambio y a la reciente marginalización de los países en desarrollo en el contexto de la economía mundial, determinaron que los países latinoamericanos hubieran de enfrentar una severa situación de estrangulamiento externo y se vieran en la necesidad de reducir sus vinculaciones con el exterior y de seguir una política de crecimiento hacia adentro, basada en la industrialización por substitución de importaciones.

La acelerada expansión económica que experimentaron los países del sistema trilateral durante el decenio siguiente a 1965 indujo a América Latina a acoplarse más estrechamente a la economía mundial, lo que se tradujo tanto en el incremento de sus intercambios comerciales como en Ios flujos de capital, y se refleja en el hecho de que la expansión de sus importaciones por cada $1 \%$ de aumento del producto se incrementó de $0.4 \%$ durante el período de 1950 1964 a $1.4 \%$ durante el período 1965-1974, lo que revela hasta qué punto había sido removido aquel escollo externo y en qué medida las economías latinoamericanas lograron integrarse en la economía mundial.

A partir de 1974 los países latinoamericanos deben enfrentar graves turbulencias externas, provocadas en buena medida por el aumento de los precios del petróleo, y traducidas en un escenario mundial crónicamente inflacionario, recesivo y proteccionista, un período durante el cual la mayoría de los países latinoamericanos procuró evitar la contracción que se habría derivado de la aplica- 
ción de las prescripciones ortodojas, mediante el recurso al endeudamiento externo, facilitado por un clima de extraordinaria permisibilidad financiera internacional.

A lo largo de estos últimos treinta años, América Latina ha dado muestras, así, de una curiosa mezcla de vulnerabilidad y de capacidad de respuesta frente al ciclo externo. Tomando en cuenta la profundidad de las transformaciones que ha experimentado la economía mundial a partir del decenio pasado, resulta particularmente importante preguntarse por el tipo de respuesta que deberá ensayar América Latina durante el período de transición e incertidumbre que caracterizará, seguramente, a los años 80 .

En todo caso, vale la pena destacar que, como corolario de ese cambiante proceso, entre 1950 y 1975 la región fue capaz de cuadruplicar su producción y de alcanzar una dimensión comparable a la de la producción europea en 1950, y se estima que hacia 1985 habrá alcanzado un volumen de producción similar a la de Europa en 1970, Io que señalaría una tendencia a acortar la brecha que separa a las dos regiones. Estos logros son más impresionantes si se atiende a sus aspectos más desagregados o cualitativos. A ló largo de esos 25 años la producción de acero se multiplicó en más de 15 veces, la de energía en 8 voces, y la de maquinarias y equipos, en 9 veces, de manera que al final del período los países latinoamericanos eran capaces de abastecer las tres cuartas partes de este tipo de demanda con producciones nacionales. En términos más generales, deben contabilizarse entre esos logros el mayor grado de desarrollo y diversificación de sus aparatos productivos, como consecuencia de la industrialización; la composición más diversificada de sus exportaciones $y$, en especial, el incremento de las de manufacturas; la potencialidad interna para producir bienes intermedios y de capital, ya señalada; el mayor acceso a fuentes de financiamiento internacional, particularmente de origen privado, y la transformación limitada, pero significativa, de la agricultura, especialmente en sus sectores modernizados.

Al mismo tiempo, se transformaron substancialmente los agentes del proceso, redefiniéndose no sólo el papel del estado y del mercado, sino también el de la empresa privada y de las corporaciones transnacionales, y el de otras instituciones que en el pasado desempeñaban roles subalternos o se movían dentro del ámbito privado, y que hoy día han pasado a tener una gravitación cada vez mayor en la evolución social de estos países.

No es de extrañar que uno de los resultados de esas transformaciones que más se ha señalado es el aumento de la capacidad de defensa de los países latinoamericanos frente a las turbulencias externas que tan agudamente se han hecho sentir en los últimos años. 
Como consecuencia de las tendencias señaladas, concluye un informe autorizado, "durante esta década se han transformado profundamente las formas de inserción de Aimérica Latina en la economía mundial y se ha intensificado el proceso de internacionalización y de vinculación con los países industriales con economía de mercado"14.

La profunda transformación económica y social registrada por los países latinoamericanos durante estos últimos decenios, y su mayor entrelazamiento con Ia economía mundial, implican un complejo balance de riesgos y oportunidades. Entre las consecuencias negativas de ese proceso se cuenta el doble impacto de las alzas del precio del petróleo y de la estanflación en los centros, con su secuela neo-proteccionista, un síndrome que afectará tanto el dinamismo de las exportaciones latinoamericanas como la evolución de sus términos de intercambio. Entre las oportunidades que Ios cambios ocurridos a nivel mundial podrían abrir a los países latinoamericanos, cabe registrar la prioridad asignada al sector de la energía y los recursos naturales, las perspectivas (si bien ambiguas) que abre el proceso de reindustrialización que habrá de encarar el Norte en los años 80, así como la forma como participe el Sur en el redespliegue industrial subsiguiente, y la evolución de los mexcados financieros internacionales, incluyendo sus perspectivas de estabilidad a largo plazo y el papel que asuman la banca privada y los organismos financieros internacionales en este proceso.

Parece imperiosa la necesidad de estudiar en profundidad las condiciones creadas por un nuevo contexto, cuyo rasgo predominante es la interdependencia, así como las nuevas formas de inserción externa que aquellas condiciones permiten -o imponen- a los paises en desarrollo. EI análisis de las relaciones externas de los países latinoamericanos --apartándose de las primeras tesis cepalinas acerca de la importancia del contexto externo para el desarrollo de nuestras naciones - ha adolecido del triple defecto de tomar como punto de partida casi exclusivamente los problemas de los países de la región en lugar de analizar con mayor profundidad las tendencias observables en los centros (de las cuales, en razón de nuestra "dependencia", somos evidentemente tributarios); de haber impreso un carácter estático al análisis del sistema internacional, de suyo escaso y, por consiguiente, de haber definido ese contexto externo bajo la forma de una sucesión de "fotografías" de carácter coyuntural que impiden ver el funcionamiento de aquellas tendencias estructurales y de largo plazo que los está transformando en su misma esencia. Los estudios que aquí se sugieren se propondrían, precisamente, superar estas omisiones.

\footnotetext{
${ }^{\text {Is }}$ GEPAL, Estudio Económico de América Latina, 1979.
} 


\section{Algunas perspectivas}

Las realidades han superado el esquema "realista" de análisis de las relaciones internacionales, fundamentalmente basado en el concepto del poder y en los objetivos correspondientes a la esfera de la seguridad, dando lugar a un mundo más interdependiente. En el estudio de las relaciones externas de los países de la periferia, aquel reduccionismo político fue reemplazado por otro de tipo económi$\mathrm{co}$, que las hizo girar en torno a una forma unilateral de dependencia externa y a un limitado número de problemas, vinculados a la evolución de los términos de intercambio entre materias primas y productos industriales, dentro de una división internacional del trabajo concebida como extremadamente rígida. Ese esquema de análisis de las relaciones centro-periferia, tanto en sus explicaciones económicas como en sus supuestos políticos, se refería a un sistema internacional que hoy se ha modificado. Es necesario intentar una reinterpretación del sistema internacional a la luz del proceso de transnacionalización que en la actualidad lo caracteriza $y$, a partir de ella, de las relaciones internacionales de los países en desarrollo.

Como se señalaba al comienzo, los estudios sobre el proceso de transnacionalización realizados hasta ahora desde una perspectiva Iatinoamericana son aún insuficientes para dar cuenta de un fenómeno tan complejo. Muchos de ellos se han concentrado en el estudio de los efectos de dicho proceso sobre las sociedades y las economías nacionales. Quienes han analizado los mecanismos internacionales a través de los cuales se irradia ese proceso, se han circunscrito preferentemente a sus aspectos económicos. En algunos casos, se han hecho importantes contribuciones al estudio de los mecanismos de transnacionalización existentes en otros sectores, tales como los medios de comunicación o los establecimientos militares, pero aún hace falta un esfuerzo orientado a mostrar la interrelación existente entre todos ellos.

Los estudios adicionales que se realicen en torno a esta problemática se distribuirán, probablemente, en dos grandes planos: el de los mecanismos de transnacionalización a nivel internacional y el de la forma en que la política exterior de los países latinoamericanos puede enfrentarse con esos mecanismos.

En el primero de los planos señalados cabe, a su vez, participar tres grandes grupos de problemas.

Primeramente, habría que examinar la evolución que han experimentado los rasgos centrales del sistema internacional contemporáneo, evolución que ha originado un sistema crecientemente transnacionalizado, incluyendo principalmente la coexistencia de múltiples centros de poder dentro de un sistema relativamente menos estratificado, el creciente compromiso de los estados nacionales con 
una amplia gama de objetivos políticos, económicos, sociales y culturales, y la inclusión en la agenda internacional de nuevos temas de interés global como el medio ambiente, los recursos naturales, la inflación, el desempleo, la internacionalización de los procesos productivos, un alto grado de fluidez financiera, la transmisión internacional de las diversas corrientes ideológicas y la fragmentación de Ios teatros de importancia estratégica. Dos aspectos deberían examinarse cuidadosamente dentro de este grupo de problemas: la transformación del concepto de lo que constituye un recurso de poder en un momento dado, y de sus usos (piénsese, por ejemplo, en la capacidad de endeudamiento externo de un país como un activo o como un pasivo), por una parte, y la transformación y proliferación de las arenas en que se desenvuelven las relaciones transnacionales, por la otra.

En segundo lugar deberían analizarse más específicamente los distintos "juegos" o "circuitos" que configuran la compleja trama de las relaciones transnacionales contemporáneas. Para no avanzar prematuramente en la identificación de esos circuitos, baste sugerir que ellos podrían agruparse en las tres categorías utilizadas más arriba, a saber, aquellos vinculados con la interdependencia ecológica (medio ambiente, energía, recursos naturales y el futuro de los recursos marinos), los que tienen relación con cuestiones de interdependencia económica (comercio, tecnología e internacionalización del ciclo productivo), y aquellos que se encuentran vinculados con la interdependencia financiera.

En el tercer grupo de problemas se deberían analizar ciertos aspectos del proceso de transnacionalización que presentan una gran especificidad sectorial. Nos referimos, en particular, a los mecanismos de transnacionalización observables en los planos político, estratégico y cultural.

El otro de los planos propuestos al iniciar este intento por desagregar las diversas facetas que plantea este fenómeno, se refiere al muy distinto peso, grado de penetración y signo que presentan los distintos circuitos anteriormente mencionados, desde el punto de vista de la política exterior de cada país latinoamericano, y viceversa, esto esto, a las distintas maneras como que la política internacional de cada país se desenvuelve en cada uno de estos juegos.

Una disgresión final. La resistencia que en muchos analistas provenientes del Tercer Mundo provoca el avance de la interdependencia, se debe a una confusión hasta ahora poco esclarecida. En efecto, este avance de la interdependencia (vis- $a$-vis a la desvinculación) entre los países en desarrollo y los desarrollados, no es sinónimo o consustancial con algunas experiencias de "apertura externa" realizadas a partir de distintas épocas y de diferentes circunstancias, a manera de opciones pretendidamente contrapuestas a las estrategias "crecimiento hacia adentro" seguidas en el pasado, expe- 
riencias que en los últimos años fueron protagonizadas por un número creciente de países latinoamericanos con diversas combinaciones de pragmatismo y ortodoxia. En otras palabras, el reconocimiento de que está cambiando aquella tendencia a la desvinculación de los países periféricos frente a los centros industriales que predominó en la postguerra y de que ella está siendo reemplazada por una mayor integración de los países en desarrollo dentro de la economía mundial, no significa renegar del "crecimiento hacia adentro" ni abrazar sin contrapesos estrategias aperturistas de crecimiento exo-dirigido. Se trata de dos planos diferentes. Sin embargo, los rasgos que objetivamente presenta el escenario internacional contemporáneo algo tiene que decir acerca de la estrategia externa que deberian adoptar los países en desarrollo, con relativa independencia del hecho de que hayan optado por un modelo "aperturista", en donde las exportaciones actúen como el "motor" del desarrollo, se hayan orientado en el sentido de un "crecimiento hacia adentro", utilizando las exportaciones como el "lubricante" de ese desarrollo, según la conocida imagen de Lewisis, o hayan ensayado algún tipo de combinación entre ambos modelos, dependiendo de su ideología y de las características de su economfa. Apuntamos aquí hacia la hipótesis de que, cualquiera que sea el camino que escoja un país en desarrollo dentro de este espectro, habrá de recorrerlo dentro de un marco caracterizado por un creciente entrelazamiento con el sistema internacional, muy diferente de la situación imperante en la postguerra.

Lo que una y otra vez se pretende subrayar en estas reflexiones es que el mejoramiento de las relaciones entre los países desarrollados y en desarrollo sólo puede concebirse dentro del marco de la evolución del sistema internacional en su conjunto. No depende, como se concebía en un comienzo, de los programas de cooperación internacional puestos en juego por los países industrializados. Tampoco depende, como se creyó más tarde, de las concesiones arrancadas a éstos por la presión de los países en desarrollo debidamente organizados. Depende, cada vez en mayor medida, de las reformas estructurales que de común acuerdo, aunque no sin enfrentamientos y presiones, se introduzcan en un sistema internacional que, para muchos efectos, ha pasado a ser uno solo.

De este énfasis se deriva, entre otras conclusiones, la noción de que el ritmo y estilo del desarrollo de los países de la periferia estará cada vez más estrechamente asociado al grado y forma que adopte su integración en el sistema transnacional. Dicha integración no es exclusivamente un "proyecto transnacionalizador" impulsado por los centros sino que, sin desestimar los intereses ni el poderío de éstos, responde a la dinámica global del sistema trans-

A. Lewis, op cit, 1980. 
nacional contemporáneo, Las consecuencias de este proceso, por otra parte, no son puramente negativas: la integración de los países en desarrollo en este sistema entraña al mismo tiempo riesgos y oportunidades para ellos. Dichos países enfrentan el desafío de encontrar un equilibrio adecuado entre los costos que podría infligirles ese proceso y los beneficios que podrían obtener de una participación más amplia, agresiva y diversificada en el sistema. De aquí se derivan múltiples corolarios con respecto a la estrategia de negociación externa que deberían seguir los países en desarrollo en un mundo interdependiente, en que la sensibilidad de las diversas sociedades nacionales frente al contexto externo se intensifica, un tema que se ha desarrollado en otro lugar recientemente ${ }^{16}$.

La sugerencia que se quiere dejar planteada aquí consiste en que la integración de los países periféricos en el sistema transnacional no tiene por qué acarrear sólo consecuencias negativas ni respondé exclusivamente a los intereses o designios de aquellos países que ocupan una posición dominante en el sistema. Se trata, por el contrario, de un proceso que presenta un margen de ambigüedad considerable desde el punto de vista de sus consecuencias para los países en desarrollo, y frente al cual éstos pueden asumir un papel más o menos activo o pasivo. Lo anterior debería inducir a esos países a rechazar tanto una estrategia de integración indiscriminada en el sistema transnacional, como una de aislamiento internacional o de "desarrollo separado", explorando las posibilidades de articular estrategias de "participación selectiva" en el sistema.

Uno de los primeros llamados en favor de una "participación se-" lectiva" en el sistema internacional se encuentra en la Declaración sobre la Situación de América Latina en la Actual Coyuntura Económica Internacional, formulada por el Foro Latinoamericano en Caracas en abril de 1975. "La experiencia latinoamericana demuestra la necesidad de abandonar la idea de que el estímulo fundamental al desarrollo proviene de una inserción total en el sistema económico internacional. Por el contrario, la implantación de nuevos estilos de consumo y producción, así como la consecución de un nivel mínimo de autonomía, requieren avanzar hacia políticas de participación selectiva en el sistema, que permitan escoger el tipo de vînculaciones que se desea tener. Se trata de minimizar la dependencia, de maximizar la autonomía y de buscar nuevos modelos de desarrollo autosostenido"17.

Tales estrategias suponen la posibilidad de combinar diversos grados de apertura y de intervención, por parte de los estados na-

${ }^{16}$ L. Tomassini, Las Negociaciones Norte-Sur: Algunas Alternativas, documento presentado en una reunión sobre el porvenir de las negociaciones globales. realizada en el CEESTEM en julio de 1981.

${ }^{1 T E s t u d i o s ~ d e l ~ T e r c e r ~ M u n d o, ~ V o l . ~ I, ~ N o ~ 1, ~ m a r z o ~ d e ~} 1978$. 
cionales, dentro de una gama de alternativas como la que se sugería en el siguiente diagrama ${ }^{18}$ :

Intervención Estatal

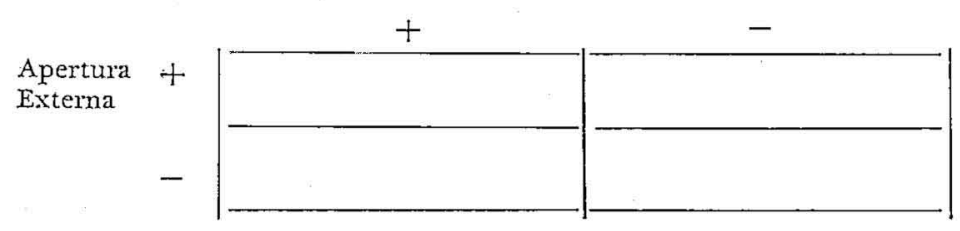

Sería posible, así, distinguir, de un modo general, cuatro tipos de estrategia externa: 1) apertura con intervención como, por ejemplo, en las experiencias de Brasil y Corea del Sur;2) apertura sin intervención, como en el caso de Chile; 3) aislamiento (o protección) con intervención, como en el caso de las estrategias de sustitución de importaciones, y otras experiencias más recientes, y 4) aislamiento sin intervención, como en las estrategias autarquizantes orientadas hacia la satisfacción de las necesidades básicas, propuestás para algunos países de menor grado de desarrollo. En esta tipología, el concepto de intervención es entendido como el ejercicio de la capacidad nacional, principalmente expresada a través del estado, para regular la aplicación de la estrategia $y$, por consiguiente, el grado y forma de inserción externa de las sociedades.

El margen de maniobra para la aplicación de uno u otro tipo de estrategia dependerá, obviamente, de las características que presente cada país, incluyendo el tamaño y grado de desarrollo de su economía, su estructura económica y social, su sistema político, su importancia estratégica y la forma que tradicionalmente han adoptado sus relaciones externas. Naturalmente, la posibilidad de controlar la forma y grado de inserción externa de una sociedad determinada $-\mathrm{y}$, por consiguiente, el grado de autonomía relativa de su respectivo proceso de desarrollo- depende estrechamente de las op: ciones que dicha sociedad adopte con respecto a las restantes dimensiones en función de las cuales es posible caracterizar su estilo de desarrollo- un tema que nos llevaría a explorar territorios más alejados de estas reflexiones ${ }^{19}$.

${ }^{15 *}$ Tomado del trabajo de O. Sunkel y L. Tomassini ya citado.

${ }^{19} \mathrm{En}$ otro lugar (O. Sunkel y L. Tomassini, op. cit.) se ha señalado que el estilo de desarrollo predominante en una sociedad determinada se puede caracterizar en función de las opciones que ésta adopte en relación con diversas dimensiones, entre las cuales se cuentan a) el crecimiento económico, b) el desarrollo social, c) la participación política, d) la identidad cultural, e) su sustentabilidad ecológica y f) su grado de autonomía nacional. En ese lugar se postulaba la existencia de una necesaria correlación entre las posiciones adoptadas por un país determinado frente a las diversas dimensiones anteriormente mencionadas. 
La posibilidad de ensayar una estrategia de participación selectiva en un sistema que presiona en favor de la integración transnacional de sus distintas unidades, en razón de su creciente interdependencia, depende en último término de la organización política de las distintas sociedades. El reconocimiento y análisis de aquella creciente relación de interdependencia no equivale necesariamente a situar el motor del desarrollo de un país en su contexto externo, ni a reducir la elección de sus formas de inserción externa a un problema predominantemente tecnocrático, ni a separar la política interna de la internacional, privilegiando la importancia de esta última. La selectividad de la estrategia externa de cada país depende de su grado de identidad nacional, manifestada en la claridad con que define sus intereses y en la firmeza con que los persigue en el plano internacional, y esta es función de su organización política. Sólo puede practicarla un país que es capaz de articular una voluntad nacional, a través de un sistema político participativo y democrático, en que no sólo se expresen las distintas dependencias del estado sino todos los sectores que integran su trama social. Los regímenes autoritarios instaurados para asegurar el predominio de determinadas minorías carecen por definición de Ia capacidad de hacerlo, porque han renunciado a interpretar los intereses mayoritarios de sus sociedades cuando no son proclives a servir los de los grupos transnacionales que los apoyan, y porque su carencia de una base consensual debilita la estabilidad de sus políticas. 